\title{
Planar Brownian flows with rank-based characteristics
}

Mine Çağlar, Abdullah Harun Karakuş, and loannis Karatzas

Citation: AIP Conference Proceedings 1978, 320005 (2018); doi: 10.1063/1.5043936

View online: https://doi.org/10.1063/1.5043936

View Table of Contents: http://aip.scitation.org/toc/apc/1978/1

Published by the American Institute of Physics

\section{Conference Proceedings}

\section{Get $30 \%$ off all print proceedings!}

Enter Promotion Code PDF-30 at checkout 


\title{
Planar Brownian Flows with Rank-Based Characteristics
}

\author{
Mine Çağlar ${ }^{1, a)}$, Abdullah Harun Karakuşş,b) and Ioannis Karatzas ${ }^{2, c)}$ \\ ${ }^{1}$ Koç University, Istanbul, Turkey \\ ${ }^{2}$ Columbia University, New York, USA

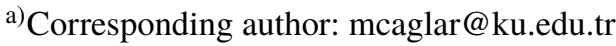 \\ b) akarakus15@ku.edu.tr \\ c)ikaratzas@icloud.com
}

Abstract. We study a stochastic differential equation with rank-based characteristics on the plane. We find its flow solutions and characterize coalescence.

\section{INTRODUCTION}

Stochastic differential equations that are driven by smooth vector fields give rise to stochastic flows when they have strong solutions. These flows are homeomorphic, meaning, when two particles governed by this flow start at different positions, they never meet. However, it is proved in [3] that when the covariance function is not smooth on the diagonal, these SDEs give rise to different stochastic flows where coalescence is possible. Moreover, as it is examined in [4], they give rise to turbulent evolutions which are represented by flows of probability kernels. In this case, we also witness splitting, the flow no longer determines the motion of a particle, but it describes how a point mass is spread.

In [1], planar diffusions with rank-based characteristics, whose dynamics are described below, have been analyzed in detail. In this study, we find the flow solutions of this rank-based stochastic differential equation, assuming there is no drift term. Using the same notation, we define

$$
\Sigma_{+}=\left(\begin{array}{cc}
\rho \cos \varphi & -\rho \sin \varphi \\
\sigma \varepsilon \sin \varphi & \sigma \varepsilon \cos \varphi
\end{array}\right) \quad \Sigma_{-}=\left(\begin{array}{cc}
\sigma \cos \vartheta & -\sigma \sin \vartheta \\
\rho \delta \sin \vartheta & \rho \delta \cos \vartheta
\end{array}\right)
$$

where $\rho, \sigma, \varepsilon, \delta \in\{-1,+1\}, 0 \leq \varphi, \vartheta \leq 2 \pi$ and we set $\Sigma\left(x_{1}, x_{2}\right)=\Sigma_{+} \mathbf{1}_{\left\{x_{1}>x_{2}\right\}}+\Sigma_{-} \mathbf{1}_{\left\{x_{1} \leq x_{2}\right\}}$. We study the following stochastic differential equation

$$
d \mathbf{X}(t)=\Sigma(\mathbf{X}(t)) d \mathbf{W}(t),
$$

where $\mathbf{W}=\left(W^{1}, W^{2}\right)^{\prime}$ is a two-dimensional standard Brownian motion, $\mathbf{X}=\left(X^{1}, X^{2}\right)^{\prime}$, and apostrophe denotes transposition. Although the matrices $\Sigma_{+}$and $\Sigma_{-}$vary the configuration of the diffusion with respect to the coordinates of $\mathbf{W}$ on $\mathbb{R}^{2}$, all choices of the parameters yield the same local covariance matrix given by

$$
\left(\begin{array}{cc}
\rho^{2} \mathbf{1}_{\left\{x_{1}>x_{2}\right\}}+\sigma^{2} \mathbf{1}_{\left\{x_{1} \leq x_{2}\right\}} & 0 \\
0 & \rho^{2} \mathbf{1}_{\left\{x_{1} \leq x_{2}\right\}}+\sigma^{2} \mathbf{1}_{\left\{x_{1}>x_{2}\right\}}
\end{array}\right) .
$$

In [1], $\rho^{2}+\sigma^{2}=1$ is assumed. However, we choose both $\rho$ and $\sigma$ to be \pm 1 so that one-point motion $\mathbf{X}$ of (1) is a two-dimensional Brownian motion.

In the following sections, we first perform a clockwise rotation to simplify the analysis and characterize the existence of strong and weak solutions. Second, we prove that the flow solutions exist and study coalescence in a case by case manner on the parameter space. 


\section{STRONG AND WEAK SOLUTIONS}

We perform a clockwise rotation on $\mathbb{R}^{2}$ by $\pi / 4$ with the matrix $R=\frac{1}{\sqrt{2}}\left(\begin{array}{cc}1 & 1 \\ -1 & 1\end{array}\right)$ and set $\tilde{\mathbf{X}}=R \mathbf{X}$, to get

$$
\begin{gathered}
d \tilde{X}_{t}^{1}=1_{\left\{\tilde{X}_{t}^{2}<0\right\}} d \tilde{B}_{t}^{1}+1_{\left\{\tilde{X}_{t}^{2} \geq 0\right\}} d \tilde{B}_{t}^{2}, \\
d \tilde{X}_{t}^{2}=1_{\left\{\tilde{X}_{t}^{2}<0\right\}} d \tilde{B}_{t}^{3}+1_{\left\{\tilde{X}_{t}^{2} \geq 0\right\}} d \tilde{B}_{t}^{4},
\end{gathered}
$$

where $R \Sigma_{+} \mathbf{W}=\left(\tilde{B}^{1}, \tilde{B}^{3}\right)^{\prime}$ and $R \Sigma_{-} \mathbf{W}=\left(\tilde{B}^{2}, \tilde{B}^{4}\right)^{\prime}$ and they are two-dimensional Brownian motions. These Brownian motions also satisfy

$$
\begin{aligned}
d\left\langle\tilde{B}^{1}, \tilde{B}^{2}\right\rangle_{t} & =\frac{1}{2}(\rho \sigma(1+\varepsilon \delta) \cos (\varphi-\vartheta)+(\varepsilon-\delta) \sin (\varphi-\vartheta)) \\
d\left\langle\tilde{B}^{3}, \tilde{B}^{4}\right\rangle_{t} & =\frac{1}{2}(\rho \sigma(1+\varepsilon \delta) \cos (\varphi-\vartheta)-(\varepsilon-\delta) \sin (\varphi-\vartheta)) \\
d\left\langle\tilde{B}^{1}, \tilde{B}^{4}\right\rangle_{t} & =\frac{1}{2}(\rho \sigma(-1+\varepsilon \delta) \cos (\varphi-\vartheta)-(\varepsilon+\delta) \sin (\varphi-\vartheta))=d\left\langle\tilde{B}^{2}, \tilde{B}^{3}\right\rangle_{t}
\end{aligned}
$$

This rotation will simplify the analysis of our problem and while proving the existence of weak and strong solutions and the flow solution we will work with (2) and (3).

We know from [1, Thm. 5.2] that Equation (1) fails to have a strong solution if and only if $\left(\mathbf{e}_{1}-\mathbf{e}_{2}\right)^{\prime} \Sigma_{-}=-\left(\mathbf{e}_{1}-\right.$ $\left.\mathbf{e}_{2}\right)^{\prime} \Sigma_{+}$, where $\mathbf{e}_{1}=(1,0)^{\prime}$ and $\mathbf{e}_{2}=(0,1)^{\prime}$. It has a unique in law weak solution for all configurations $\Sigma$ [1]. We have the following equivalent characterization of weak and strong existence, by noting that (3) is the Tanaka equation if and only if $\left(\mathbf{e}_{1}-\mathbf{e}_{2}\right)^{\prime} \Sigma_{-}=-\left(\mathbf{e}_{1}-\mathbf{e}_{2}\right)^{\prime} \Sigma_{+}$.

Proposition 1. Equation (1) fails to have a strong solution if and only if Equation (3) is the Tanaka equation.

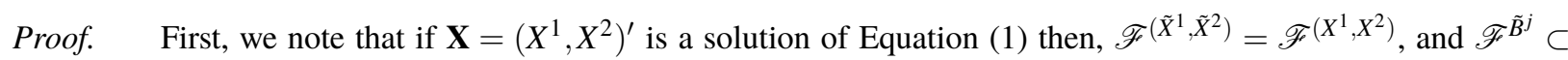
$\mathscr{F}^{\left(W^{1}, W^{2}\right)}$ holds for $j=1,2,3,4$. If (3) is not the Tanaka equation then, either $d\left\langle\tilde{B}^{3}, \tilde{B}^{4}\right\rangle_{t}=1$ or $\left|d\left\langle\tilde{B}^{3}, \tilde{B}^{4}\right\rangle_{t}\right|<1$. In the first case, it trivially has a strong solution. In the second case, it has a strong solution by [2, Thm. 1.1]. Therefore the relation $\mathscr{F} \tilde{X}^{2} \subset \mathscr{F}\left(\tilde{B}^{3}, \tilde{B}^{4}\right)$ holds. It is also clear that the relation $\mathscr{F} \tilde{X}^{1} \subset \mathscr{F}\left(\tilde{X}^{2}, \tilde{B}^{1}, \tilde{B}^{2}\right)$ holds, too. It follows that $\mathscr{F}\left(X^{1}, X^{2}\right) \subset \mathscr{F}^{\left(W^{1}, W^{2}\right)}$. We first note that $\mathscr{F}^{\left(W^{1}, W^{2}\right)}=\mathscr{F}^{\left(\tilde{B}^{1}, \tilde{B}^{3}\right)}$. Now, assume that (3) is the Tanaka equation, that is, $d\left\langle\tilde{B}^{3}, \tilde{B}^{4}\right\rangle_{t}=-1$, so that $\mathscr{F}^{\tilde{B}^{3}} \subsetneq \mathscr{F}^{2}$ and (1) has a strong solution. Then, we have the following relations

$$
\mathscr{F}^{\left(\tilde{X}^{2}, \tilde{B}^{1}\right)} \subset \mathscr{F}^{\left(W^{1}, W^{2}\right)}=\mathscr{F}^{\left(\tilde{B}^{3}, \tilde{B}^{1}\right)} \subsetneq \mathscr{F}^{\left(\tilde{X}^{2}, \tilde{B}^{1}\right)},
$$

which is a contradiction.

\section{FLOW AND COALESCENCE}

A flow solution of (1) is a stochastic flow of mappings $\varphi=\left(\varphi^{1}, \varphi^{2}\right)$ [4, Definition 1.6]. That is, $\left\{\varphi_{s, t}, s \leq t\right\}$ is a family of random variables, and for all $s \leq t, \varphi_{s, t}$ is a measurable mapping from $\left(\mathbb{R}^{2} \times \Omega, \mathscr{B}\left(\mathbb{R}^{2}\right) \otimes \mathscr{A}\right)$ to $\left(\mathbb{R}^{2}, \mathscr{B}\left(\mathbb{R}^{2}\right)\right)$ satisfying

$$
d \varphi_{s, t}=\Sigma\left(\varphi_{s, t}\right) d W_{s, t},
$$

where $\mathbf{W}_{s, t}=\left(W_{s, t}^{1}, W_{s, t}^{2}\right)^{\prime}$ is a white noise, or equivalently,

$$
\begin{aligned}
& \varphi_{s, t}^{1}(x)=x_{1}+\int_{s}^{t} 1_{\left\{\varphi_{s, u}^{1}>\varphi_{s, u}^{2}\right\}} d B_{s, u}^{1}+\int_{s}^{t} 1_{\left\{\varphi_{s, u}^{1} \leq \varphi_{s, u}^{2}\right\}} d B_{s, u}^{2}, \\
& \varphi_{s, t}^{1}(x)=x_{2}+\int_{s}^{t} 1_{\left\{\varphi_{s, u}^{1}>\varphi_{s, u}^{2}\right\}} d B_{s, u}^{3}+\int_{s}^{t} 1_{\left\{\varphi_{s, u}^{1} \leq \varphi_{s, u}^{2}\right\}} d B_{s, u}^{4},
\end{aligned}
$$

where $\left(B^{1}, B^{3}\right)^{\prime}=\Sigma_{+} W$ and $\left(B^{2}, B^{4}\right)^{\prime}=\Sigma_{-} W$. We also have $R\left(B^{1}, B^{3}\right)^{\prime}=\left(\tilde{B}^{1}, \tilde{B}^{3}\right)^{\prime}$ and $R\left(B^{2}, B^{4}\right)^{\prime}=\left(\tilde{B}^{2}, \tilde{B}^{4}\right)^{\prime}$. 
There are three cases for $\tilde{B}^{3}$ and $\tilde{B}^{4}$ in $(3) ; d\left\langle\tilde{B}^{3}, \tilde{B}^{4}\right\rangle_{t}$ is equal to $1,-1$, or a value between the two. In the first case, it trivially has a flow solution since it is a one-dimensional Brownian motion. In the second and third cases, we know from [2, Thm. 1.1], [5, Thm. 1], [3, Prop. 7.1], and [4, Example 4.4.3] that Equation (3) has a flow solution. On the other hand, the right hand side of Equation (2) does not depend on $\tilde{X}^{1}$ so we can always integrate it, after $\tilde{X}^{2}$ is solved for. Therefore, the equations for a flow solution $\tilde{\varphi}$ of (2) and (3) are

$$
\begin{aligned}
\tilde{\varphi}_{s, t}^{1}\left(\tilde{x}_{1}, \tilde{x}_{2}\right) & =\tilde{x}_{1}+\int_{s}^{t} 1_{\left\{\tilde{\varphi}_{s, u}^{2}\left(\tilde{x}_{2}\right)<0\right\}} d \tilde{B}_{s, u}^{1}+\int_{s}^{t} 1_{\left\{\tilde{\varphi}_{s, u}^{2}\left(\tilde{x}_{2}\right) \geq 0\right\}} d \tilde{B}_{s, u}^{2}, \\
\tilde{\varphi}_{s, t}^{2}\left(\tilde{x}_{2}\right) & =\tilde{x}_{2}+\int_{s}^{t} 1_{\left\{\tilde{\varphi}_{s, u}^{2}\left(\tilde{x}_{2}\right)<0\right\}} d \tilde{B}_{s, u}^{3}+\int_{s}^{t} 1_{\left\{\tilde{\varphi}_{s, u}^{2}\left(\tilde{x}_{2}\right) \geq 0\right\}} d \tilde{B}_{s, u}^{4} .
\end{aligned}
$$

Setting $\varphi_{s, t}=R^{-1} \tilde{\varphi}_{s, t}$ we see that Equations (4) and (5) are satisfied. As a result, we have the following theorem since $\tilde{\varphi}$ is a flow and $R^{-1}$ is a deterministic rotation.

Theorem 2. There exists a flow solution of Equation (1) given by $\varphi=R^{-1} \tilde{\varphi}$.

Given two solutions $X$ and $Y$ of (1) starting at different positions $x \in \mathbb{R}^{2}$ and $y \in \mathbb{R}^{2}$ respectively, if we can find a stopping time $T$ which is finite with positive probability and satisfies $X_{T}=Y_{T}$, then we say the flow is coalescing. We study coalescence because as it is proved in [4], all kernel solutions of the SDE can be found by filtering the coalescing solution. We have $X_{T}=Y_{T}$ if and only if $\tilde{X}_{T}=\tilde{Y}_{T}$ where $\tilde{X}$ and $\tilde{Y}$ start at $R x=\tilde{x}$ and $R y=\tilde{y}$ respectively. Therefore, we will study coalescence for the system of stochastic differential equations in (2) and (3), instead of (1).

The Brownian motions in (2) and (3) have the following cross variations

$$
\begin{aligned}
d\left\langle\tilde{B}^{1}, \tilde{B}^{2}\right\rangle_{t} & =\frac{1}{2}(\rho \sigma(1+\varepsilon \delta) \cos (\varphi-\vartheta)+(\varepsilon-\delta) \sin (\varphi-\vartheta)), \\
d\left\langle\tilde{B}^{3}, \tilde{B}^{4}\right\rangle_{t} & =\frac{1}{2}(\rho \sigma(1+\varepsilon \delta) \cos (\varphi-\vartheta)-(\varepsilon-\delta) \sin (\varphi-\vartheta)) .
\end{aligned}
$$

We study coalescence in two broad categories, namely $\varepsilon=-\delta$ and $\varepsilon=\delta$.

\section{The Case $\varepsilon=-\delta$}

In this case, the cross variation processes in (8) and (9) simplify to

$$
d\left\langle\tilde{B}^{1}, \tilde{B}^{2}\right\rangle_{t}=\varepsilon \sin (\varphi-\vartheta) \quad d\left\langle\tilde{B}^{3}, \tilde{B}^{4}\right\rangle_{t}=-\varepsilon \sin (\varphi-\vartheta)
$$

Now, we have three possibilities: $\varepsilon \sin (\varphi-\vartheta)=1, \varepsilon \sin (\varphi-\vartheta)=-1$, or $|\sin (\varphi-\vartheta)|<1$.

If $\varepsilon \sin (\varphi-\vartheta)=1$, then the flow $\tilde{\varphi}^{1}$ and $\tilde{\varphi}^{2}$ satisfy (6) and (7), which turn into

$$
\begin{aligned}
& \tilde{\varphi}_{s, t}^{1}\left(\tilde{x}_{1}\right)=\tilde{x}_{1}+\int_{s}^{t} d \tilde{B}_{s, u}^{1}, \\
& \tilde{\varphi}_{s, t}^{2}\left(\tilde{x}_{2}\right)=\tilde{x}_{2}+\int_{s}^{t} \operatorname{sgn}\left(\tilde{\varphi}_{s, u}^{2}\right) d \tilde{B}_{s, u}^{3} .
\end{aligned}
$$

Starting with two different initial positions $\left(\tilde{x}_{1}, \tilde{x}_{2}\right)$ and $\left(\tilde{y}_{1}, \tilde{y}_{2}\right)$, the two particles evolve according to this flow equation. Note that the difference $\tilde{\varphi}_{s, t}^{1}\left(\tilde{x}_{1}\right)-\tilde{\varphi}_{s, t}^{1}\left(\tilde{y}_{1}\right)=\tilde{x}_{1}-\tilde{y}_{1}$ is fixed. Also, it is known that given $\tilde{x}_{2}, \tilde{y}_{2}$ there exists an almost surely finite stopping time $T$ such that $\tilde{\varphi}_{s, T}^{2}\left(\tilde{x}_{2}\right)=\tilde{\varphi}_{s, T}^{2}\left(\tilde{y}_{2}\right)\left[5\right.$, Thm. 1], [3, Prop. 7.1], [4, Example 4.4.3]. If $\tilde{x}_{1}=\tilde{y}_{1}$ then, we also have $\tilde{\varphi}_{s, T}^{1}\left(\tilde{x}_{1}\right)=\tilde{\varphi}_{s, T}^{1}\left(\tilde{y}_{1}\right)$. Therefore, we have coalescence if and only if $\tilde{x}_{1}=\tilde{y}_{1}$, that is, $x_{1}+x_{2}=y_{1}+y_{2}$.

Similarly, if $\varepsilon \sin (\varphi-\vartheta)=-1$, then the flow solutions $\tilde{\varphi}^{1}$ and $\tilde{\varphi}^{2}$ satisfy

$$
\begin{aligned}
& \tilde{\varphi}_{s, t}^{1}\left(\tilde{x}_{1}\right)=\tilde{x}_{1}+\int_{s}^{t} \operatorname{sgn}\left(\tilde{\varphi}_{s, u}^{2}\right) d \tilde{B}_{s, u}^{1}, \\
& \tilde{\varphi}_{s, t}^{2}\left(\tilde{x}_{2}\right)=\tilde{x}_{2}+\int_{s}^{t} d \tilde{B}_{s, u}^{3} .
\end{aligned}
$$

Note that given $\tilde{x}_{2}$ and $\tilde{y}_{2}$ the difference $\tilde{\varphi}_{s, t}^{2}\left(\tilde{x}_{2}\right)-\tilde{\varphi}_{s, t}^{2}\left(\tilde{y}_{2}\right)=\tilde{x}_{2}-\tilde{y}_{2}$ is fixed. Then, for any possibility of coalescence we need $\tilde{x}_{2}=\tilde{y}_{2}$. However, this implies that $\tilde{\varphi}_{s, t}^{1}\left(\tilde{x}_{1}\right)-\tilde{\varphi}_{s, t}^{1}\left(\tilde{y}_{1}\right)=\tilde{x}_{1}-\tilde{y}_{1}$ for any $\tilde{x}_{1}$ and $\tilde{y}_{1}$. But if we also assume that 
$\tilde{x}_{1}=\tilde{y}_{1}$, then the two particles will start at the same position. Hence, if two particles start at different positions we know that they will not meet. Therefore, there is no coalescence.

The case $|\sin (\varphi-\vartheta)|<1$ remains to be studied as future work.

\section{The Case $\varepsilon=\delta$}

In this case, the cross variation processes in (8) and (9) simplify to

$$
d\left\langle\tilde{B}^{1}, \tilde{B}^{2}\right\rangle_{t}=d\left\langle\tilde{B}^{3}, \tilde{B}^{4}\right\rangle_{t}=\rho \sigma \cos (\varphi-\vartheta)
$$

Again, we have three possibilities: $\rho \sigma \cos (\varphi-\vartheta)=1, \rho \sigma \cos (\varphi-\vartheta)=-1$, or $|\cos (\varphi-\vartheta)|<1$.

If $\rho \sigma \cos (\varphi-\vartheta)=1$, then this suggests that $\tilde{B}^{1}=\tilde{B}^{2}$ and $\tilde{B}^{3}=\tilde{B}^{4}$, so given two solutions of (2) and (3) the distance between them will be fixed at all times. Therefore, there is no coalescence.

The cases i) $\rho \sigma \cos (\varphi-\vartheta)=-1$ and ii) $|\cos (\varphi-\vartheta)|<1$ need to be analyzed as future work.

Acknowledgements. This work is supported by TUBITAK Project No. 115F086.

\section{References}

[1] Fernholz, E. R., Ichiba, T., Karatzas, I., Prokaj, V. (2012) Planar diffusions with rank-based characteristics and perturbed Tanaka equation, Probab. Theory Relat. Fields 156: 343-374.

[2] Çă̆lar, M., Hajri, H., Karakuş, A.H., (2017) Correlated coalescing Brownian flows on $\mathbb{R}$ and the circle. Submitted.

[3] Le Jan, Y. and Raimond, O. (2002) Integration of Brownian vector fields, Ann. Probab. Vol. 30, No. 2, 826873.

[4] Le Jan, Y. and Raimond, O. (2004) Flows, coalescence and noise, Ann. Probab. 32: 1247-1315.

[5] Watanabe, S. (2000) Stochastic flow and noise associated with the Tanaka stocahstic differential equation, Ukranian Mathematical Journal Vol. 52, No. 9. 